\title{
Iterative approach to the Schwinger variational principle applied to electron-molecular-ion collisions
}

\author{
Robert R. Lucchese and Vincent McKoy \\ Arthur Amos Noyes Laboratory of Chemical Physics, California Institute of Technology, Pasadena, California 91125
}

(Received 27 May 1980)

\begin{abstract}
We present a study of electron-molecular-ion collisions. The scattering equations are solved using an iterative approach to the Schwinger variational principle. These equations are formulated using the Coulomb Green's function to properly treat the long-range Coulomb tail of the molecular-ion potential. We apply this approach to electron-hydrogen-molecular-ion collisions in the static-exchange approximation. We obtain elastic differential cross sections, and also use the continuum states from these calculations to compute the photoionization cross section of the hydrogen molecule. The iterative method used here converged rapidly in all calculations performed.
\end{abstract}

\section{INTRODUCTION}

Considerable effort has been devoted to the development of efficient and accurate methods for solving the electron-molecule-collision problem. ${ }^{1}$ The main difficulties encountered in solving this problem are the nonspherical nature of the potential and the accurate treatment of the nonlocal exchange potential. Our approach to the solution of the electron-molecule-collision problem is to use the Schwinger variational principle. The first application of the Schwinger variational principle to this problem was an approximate discrete basis function approach. ${ }^{2}$ We then implemented the Schwinger variational principle exactly using numerical techniques. ${ }^{3.4}$ This method has been successfully applied to the scattering of low-energy electrons by $\mathrm{He}, \mathrm{He}^{+}, \mathrm{H}_{2}, \mathrm{H}_{2}{ }^{+}, \mathrm{N}_{2}{ }^{+}$, and $\mathrm{LiH}^{3-9}$ In the present paper we give results for the $e^{-}-\mathrm{H}_{2}^{+}$ system ${ }^{7}$ using a recently developed iterative technique based upon the Schwinger variational principle. $^{6}$

In this study of the $e^{-}-\mathrm{H}_{2}{ }^{+}$scattering system we make several standard simplifying assumptions. First we work within the fixed-nuclei approximation. We also assume that the interaction between the continuum electron and the molecular ion is described by the static-exchange potential, and hence we neglect electron correlation.

The iterative method used here ${ }^{6}$ for solving the resulting scattering equations begins by exactly solving the equations for a separable approximation to the static-exchange potential. The separable approximation used in this study is constructed from a set of Cartesian-Gaussian functions. The iterative method then proceeds by using the exact solutions to the approximate separable potential in a distorted-wave Schwinger variational calculation on the difference potential (i.e., the difference between the exact static-exchange potential and the approximate separable potential). Further iterations proceed to give higher-order corrections.

We present converged results for both elastic $e^{-}-\mathrm{H}_{2}^{+}$scattering and photoionization cross sections of $\mathrm{H}_{2}$. We found that our iterative method converged rapidly in all calculations presented here. We have studied $e-\mathrm{H}_{2}{ }^{+}$scattering as the first test case for applying the iterative Schwinger method to electron-molecular-ion scattering. We have chosen this system since standard singlecenter expansion methods should work well and thus provide us with accurate results to compare with. We have compared our results with the accurate static-exchange results of Collins and Robb, ${ }^{10}$ which were obtained using such a singlecenter expansion method. There have been other studies of $e^{-}-\mathrm{H}_{2}{ }^{+}$system, ${ }^{11-13}$ but the study of Collins and Robb ${ }^{10}$ is the most accurate to date. For all channels and energies considered here, the results of the iterative Schwinger variational method are in good agreement with those of Collins and Robb. ${ }^{10}$

\section{THEORY}

The Schrödinger equation for electron-molecular-ion scattering in the static-exchange approximation is (in atomic units)

$$
\left(-\frac{1}{2} \nabla^{2}-\frac{Z}{r}+V(\overrightarrow{\mathrm{r}})-\frac{k^{2}}{2}\right) \Psi_{\overrightarrow{\mathrm{k}}}^{(+)}(\overrightarrow{\mathrm{r}})=0,
$$

where $Z$ is the net charge on the isolated ion and $V(\overrightarrow{\mathrm{r}})$ is the residual short-range potential. This Schrödinger equation is equivalent to the Lippmann-Schwinger equation

$$
\Psi_{\overrightarrow{\mathrm{k}}}^{(+)}=\Psi_{\overrightarrow{\mathrm{k}}}^{c(+)}+G^{c(+)} U \Psi_{\overrightarrow{\mathrm{k}}}^{(+)},
$$

where

$$
U(\overrightarrow{\mathrm{r}})=2 V(\overrightarrow{\mathrm{r}})
$$

and the Coulomb Green's function is defined by 


$$
G^{c( \pm)}=\left(\nabla^{2}+\frac{2 Z}{r}+k^{2} \pm i \epsilon\right)^{-1}
$$

The function $\Psi_{\vec{k}}^{c(t)}$ is the pure Coulomb scattering function and is given in terms of its partial-wave expansion as

$$
\Psi_{\overrightarrow{\mathrm{k}}}^{c(t)}(\overrightarrow{\mathrm{r}})=\left(\frac{2}{\pi}\right)^{1 / 2} \sum_{l, m} i^{l} \phi_{k l m}^{c( \pm)}(\overrightarrow{\mathrm{r}}) Y_{l m}^{*}(\hat{k}),
$$

where $\phi_{k l m}^{c(t)}$ is the partial-wave Coulomb function defined by

$$
\phi_{k l m}^{c(t)}(\overrightarrow{\mathrm{r}})=e^{( \pm) i \sigma_{l}} \frac{F_{l}(\gamma ; k r)}{k r} Y_{l m}(\hat{r}) .
$$

The function $F_{l}(\gamma ; k r)$ is the regular Coulomb function with $\gamma=-Z / k$ and $\sigma_{l}$ is the Coulomb phase shift defined as $\sigma_{l}=\arg [\Gamma(l+1+i \gamma)]$.

The wave function $\Psi_{\vec{k}}^{(+)}$, which has incoming waves with momentum $\vec{k}$, can be expanded in the partial-wave series

$$
\Psi_{\vec{k}}^{(+)}(\overrightarrow{\mathrm{r}})=\left(\frac{2}{\pi}\right)^{1 / 2} \sum_{l m} i^{l} \psi_{k l m}^{(+)}(\overrightarrow{\mathrm{r}}) Y_{l m}^{*}(\hat{k}) .
$$

Computing the wave function in the partial-wave form allows the dependence of the scattering solution on the target orientation to be treated analytically. The Lippmann-Schwinger equation for the partial-wave states is then

$$
\psi_{k l m}^{(+)}(\overrightarrow{\mathrm{r}})=\phi_{k l m}^{c(+)}(\overrightarrow{\mathrm{r}})+\left\langle\overrightarrow{\mathrm{r}}\left|G^{c(+)} U\right| \psi_{k l m}^{(+)}\right\rangle
$$

Instead of solving for the scattering solutions directly, one can equivalently solve for the $T$ matrix due to only the short-range component of the potential which satisfies the Lippmann-Schwinger equation

$$
T=U+U G^{c(+)} T .
$$

Then using the identity

$$
T\left|\phi_{k l m}^{c(+)}\right\rangle=U\left|\psi_{k l m}^{(+)}\right\rangle
$$

and Eq. (8), the partial-wave solutions are obtained from

$$
\psi_{k l m}^{(+)}(\overrightarrow{\mathrm{r}})=\left\langle\overrightarrow{\mathrm{r}}\left|1+G^{c(+)} T\right| \phi_{k l m}^{c(+)}\right\rangle .
$$

We solve the Lippmann-Schwinger equation, Eq. (8), with an iterative procedure based upon the Schwinger variational principle. ${ }^{6}$ The iterative method begins by approximating the short-range potential by a separable potential of the form

$$
\left\langle\overrightarrow{\mathrm{r}}\left|U^{s} 0\right| \overrightarrow{\mathrm{r}}^{\prime}\right\rangle=\sum_{\alpha_{i}, \alpha_{j} \in R}\left\langle\overrightarrow{\mathrm{r}}|U| \alpha_{i}\right\rangle\left[U^{-1}\right]_{i j}\left\langle\alpha_{j}|U| \overrightarrow{\mathrm{r}}^{\prime}\right\rangle,
$$

where $R$ is some initial set of expansion functions. For this approximate separable potential, the solution of the Lippmann-Schwinger equation for the $T$ matrix [Eq. (9)] is given by

$$
\left\langle\overrightarrow{\mathrm{r}}\left|T^{s} 0\right| \overrightarrow{\mathrm{r}}^{\prime}\right\rangle=\sum_{\alpha_{i}, \alpha_{j} \in R}\left\langle\overrightarrow{\mathrm{r}}|U| \alpha_{i}\right\rangle\left[\left(D^{(+)}\right)^{-1}\right]_{i j}\left\langle\alpha_{j}|U| \overrightarrow{\mathrm{r}}^{\prime}\right\rangle
$$

where

$$
D_{i j}^{(+)}=\left\langle\alpha_{i}\left|U-U G^{c(+)} U\right| \alpha_{j}\right\rangle .
$$

As has been pointed out by several authors, ${ }^{14-16}$ this form of the $T$ matrix is equivalent to that obtained from finding stationary values of the Schwinger variational expression

$$
\begin{aligned}
T_{l l^{\prime} m} & =\left\langle\phi_{k l m}^{c(-)}|T| \phi_{k l^{\prime} m}^{c(+)}\right\rangle \\
& =\frac{\left\langle\phi_{k l m}^{c(-)}|U| \psi_{k l^{\prime} m}^{(+) t}\right\rangle\left\langle\psi_{k l_{m}}^{(-)}|U| \phi_{k l^{\prime} m}^{c(+)}\right\rangle}{\left\langle\psi_{k l m}^{(-) t}\left|U-U G^{c(+)} U\right| \psi_{k l^{\prime} m}^{(+) t}\right\rangle}
\end{aligned}
$$

by varying the partial-wave trial functions which are linear combinations of the expansion functions

$$
\psi_{k l m}^{(t) t}(\overrightarrow{\mathrm{r}})=\sum_{\alpha_{i} \in R} C_{k l m, i}^{(t)} \alpha_{i}(\overrightarrow{\mathrm{r}}) .
$$

The scattering solutions $\psi_{k l m}^{(+) s_{0}}$ corresponding to the approximate separable potential $U^{s_{0}}$, are obtained using Eq. (11), giving

$$
\psi_{k l m}^{(+)} s_{0}(\overrightarrow{\mathrm{r}})=\phi_{k l m}^{c(+)}(\overrightarrow{\mathrm{r}})+\left\langle\overrightarrow{\mathrm{r}}\left|G^{c(+)} T^{s_{0}}\right| \phi_{k l m}^{c(+)}\right\rangle .
$$

The iterative procedure is continued by augmenting the expansion set $R$ of Eq. (12) by the set of functions

$$
S_{0}=\left\{\psi_{k l_{1} m}^{s_{0}}, \psi_{k l_{2} m}^{s_{0}}, \ldots, \psi_{k l_{p} m}^{s_{0}}\right\}
$$

which are the scattering solutions given by- Eq. (17). Using this augmented set of functions, the first iteration is completed by calculating a new $T$ matrix given by

$$
\begin{gathered}
\left\langle\overrightarrow{\mathbf{r}}\left|T^{s_{1}}\right| \overrightarrow{\mathbf{r}}^{\prime}\right\rangle=\sum_{\chi_{i}, \chi_{j} \in R \cup s_{0}}\left\langle\overrightarrow{\mathbf{r}}|U| \chi_{i}\right\rangle\left[\left(D^{(+)}\right)^{-1}\right]_{i j} \\
\times\left\langle\chi_{j}|U| \overrightarrow{\mathbf{r}}^{\prime}\right\rangle .
\end{gathered}
$$

Note that the variational basis set $R \cup S_{0}$ used in Eq. (19) contains both the initial expansion set $R=\left\{\alpha_{i}\right\}$ and the continuum solutions given by Eq. (17). Thus, for example, the $D_{i j}^{(+)}$matrix will contain matrix elements of the form $\left\langle\psi_{k l m}^{s_{0}}\right| U$ $-U G^{c(+)} U\left|\alpha_{j}\right\rangle$ and $\left\langle\psi_{k l m}^{S_{0}}\left|U-U G^{c(+)} U\right| \psi_{k l^{\prime} m}^{S_{0}}\right\rangle$ as well as the type given in Eq. (14).

A second iteration is begun by constructing the set of solutions $S_{1}=\left\{\psi_{k l_{1} m}^{s_{1}}, \ldots, \psi_{k l_{p} m}^{s_{1}}\right\}$ which is associated with the matrix $T^{S_{1}}$ given by Eq. (19). The set $S_{1}$, combined with the initial trial function set $R$, yields a new $T$ matrix $T^{S_{2}}$. In general, $T^{S_{n}}$ and the set of functions $S_{n}$ are given by

$$
\begin{aligned}
\left\langle\overrightarrow{\mathbf{r}}\left|T^{s_{n}}\right| \overrightarrow{\mathbf{r}}^{\prime}\right\rangle=\sum_{\chi_{i}, \chi_{j} \in R \cup s_{n-1}} & \left\langle\overrightarrow{\mathbf{r}}|U| \chi_{i}\right\rangle\left[\left(D^{(+)}\right)^{-1}\right]_{i j} \\
& \times\left\langle\chi_{j}|U| \overrightarrow{\mathbf{r}}^{\prime}\right\rangle
\end{aligned}
$$


$S_{n}=\left\{\psi_{k l_{1} m, \ldots,}^{S_{n}} \psi_{k l_{p} m}^{S_{n}}\right\}$,

where

$$
\psi_{k l_{i}{ }^{m}}^{(+)} S_{n}(\overrightarrow{\mathbf{r}})=\phi_{k l_{i}{ }^{m}}^{c(+)}(\overrightarrow{\mathbf{r}})+\left\langle\overrightarrow{\mathbf{r}}\left|G^{c(+)} T^{S_{n}}\right| \phi_{k l_{i} m}^{c(+)}\right\rangle .
$$

This iterative procedure is continued until the wave functions converge. When the wave functions do converge, it can be shown that they are solutions of the Lippmann-Schwinger equation for the exact potential $U .^{6}$

In the fixed-nuclei approximation, the differential cross section (DCS) averaged over molecular

orientation is given by ${ }^{12}$

$$
\begin{aligned}
\frac{d \sigma}{d \Omega}= & \frac{\gamma^{2}}{4 k^{2} \sin ^{4}\left(\frac{\theta}{2}\right)}+\sum_{L} A_{L} P_{L}(\cos \theta) \\
& +\frac{1}{\sin ^{2}\left(\frac{\theta}{2}\right)} \sum_{L} B_{L}(\theta) P_{L}(\cos \theta),
\end{aligned}
$$

where

$$
\begin{aligned}
A_{L}=\frac{1}{4 \pi} \sum_{l, l^{\prime}, \lambda, \lambda^{\prime}, m, \mu} & a_{l l^{\prime} m} a_{\lambda \lambda^{\prime} \mu}^{*}\left(\frac{2 L+1}{2 \lambda^{\prime}+1}\right)\left(\frac{2 l+1}{2 \lambda+1}\right)^{1 / 2} \\
& \times(L l 00 \mid \lambda 0)\left(L l^{\prime} 00 \mid \lambda^{\prime} 0\right) \\
& \times(L l \mu-m m \mid \lambda \mu)\left(L l^{\prime} \mu-m m \mid \lambda^{\prime} \mu\right)
\end{aligned}
$$

and

$$
\begin{aligned}
B_{L}(\theta)=-\frac{\gamma}{k} \sum_{m} & \frac{1}{[4 \pi(2 l+1)]^{1 / 2}} \\
& \times \operatorname{Re}\left\{\exp \left[i \gamma \ln \left(\sin ^{2} \frac{\theta}{2}\right)-2 i \sigma_{0}\right] \times a_{L L m}\right\},
\end{aligned}
$$

and where $\left(j_{1} j_{2} m_{1} m_{2} \mid j m\right)$ is a Clebsch-Gordan coefficient. The fixed-nuclei dynamical coefficients $a_{l l^{\prime} m}$ are defined by ${ }^{12}$

$$
a_{l l^{\prime} m}=-\frac{\pi^{3 / 2}}{k} i^{l^{\prime-}-l}\left(2 l^{\prime}+1\right)^{1 / 2} T_{l l^{\prime} m} .
$$

In the present study, the partial-wave $T$-matrix elements are approximated at the $n$th iteration by $T_{l l^{\prime} m}^{S_{n}}$ which is given by

$T_{l l^{\prime} m}^{S_{n}}=\sum_{\chi_{i}, \chi_{j} \in R \cup s_{n-1}}\left\langle\phi_{k l m}^{c(-y}|U| \chi_{i}\right\rangle\left[\left(D^{(+)}\right)^{-1}\right]_{i j}\left\langle\chi_{j}|U| \phi_{k l m}^{c(+)}\right\rangle$

From Eq. (23) it is clearly seen that the DCS is the sum of the pure Coulomb scattering DCS plus the DCS due to the short-range potential plus an interference term.

We have only considered the interaction between the scattered electron and the target in the static- exchange approximation. The static-exchange potential due to a one-electron target is of the form ${ }^{4}$

$U(\overrightarrow{\mathrm{r}})=2\left[N^{\overrightarrow{\mathrm{A}}}(\overrightarrow{\mathrm{r}})+J^{w^{0}} \pm\left(K^{u^{\rho}}+Q^{u 0}\right) \mp\left(\epsilon_{0}+\frac{1}{2} k^{2}\right) P^{u^{0}}\right]$,

where $u^{0}(\overrightarrow{\mathrm{r}})$ is the orbital of the bound electron, and where the upper (lower) sign is for singlet (triplet) scattering. In Eq. (28), $N^{\vec{A}}$ is the nuclear attraction term minus the long-range $-Z / r$ term in Eq. (1). The operators $J^{u^{0}}$ and $K^{u^{0}}$ are the standard Coulomb and exchange operators of the orbital $u^{0}$. The $Q^{u^{0}}$ and $P^{u^{0}}$ operators are included to allow for possible nonorthogonality between the bound orbital and the continuum orbital. These operators are defined by

$$
\left\langle\overrightarrow{\mathbf{r}}\left|Q^{u^{0}}\right| \chi\right\rangle=\left\langle\overrightarrow{\mathbf{r}} \mid u^{0}\right\rangle\left\langle u^{0}|h| \chi\right\rangle+\left\langle\overrightarrow{\mathbf{r}}|h| u^{0}\right\rangle\left\langle u^{0} \mid \chi\right\rangle
$$

and

$$
\left\langle\overrightarrow{\mathrm{r}}\left|P^{u^{0}}\right| \chi\right\rangle=\left\langle\overrightarrow{\mathrm{r}} \mid u^{0}\right\rangle\left\langle u^{0} \mid \chi\right\rangle,
$$

where

$$
h(i)=-\frac{1}{2} \nabla_{i}^{2}+N^{\overrightarrow{\mathrm{A}}}\left(\overrightarrow{\mathbf{r}}_{i}\right)-Z / r_{i} .
$$

The one electron energy $\epsilon_{0}$ of the orbital $u^{0}$ is thus given by

$$
\epsilon_{0}=\left\langle u^{0}|h| u^{0}\right\rangle \text {. }
$$

\section{RESULTS FOR $e^{-}-\mathrm{H}_{2}{ }^{+}$SCATTERING}

In the present study of $e^{-}-\mathrm{H}_{2}^{+}$elastic scattering, we used a target molecular-orbital constructed from the $8 s 4 z$ Cartesian-Gaussian basis set which is given in Table $\mathrm{I}$. This basis set is the $6 s$ Gaussian fit to the hydrogen $1 s$ function given by Huzinaga, ${ }^{17}$ augmented by four $z$ functions and two diffuse $s$ functions. The internuclear separation for $\mathrm{H}_{2}^{+}$was $R=2.0$ a.u. The target energy in this basis was $E=-1.102292$ a.u. and the quadrupole moment was -1.533 a.u.

The initial scattering basis set, set $R$, is given in Table II. For the present study, $R$ consisted of a set of nuclear and bond mid-point-centered Cartesian-Gaussian functions. For all symmetries considered, the scattering basis set consisted of only five functions. We found that inclusion of bond mid-point-centered functions in the initial scattering set yielded more rapidly converging wave functions than those obtained starting from basis sets not containing such functions. We believe that this is due to the way the short-range potential $U$ as given in Eq. (28) is constructed. The short-range potential $U$ is obtained from the full static-exchange potential by the addition of the term $Z / r$. This just cancels the long-range tail of the full potential, but this method thus makes $U$ strongly repulsive near the origin. It seems that in order to describe the scattering due to this repulsive potential it is important to have 
TABLE I. Target wave function for $\mathrm{H}_{2}{ }^{+}{ }^{\mathrm{a}}$

\begin{tabular}{ccc}
\hline \hline Type of function $^{b}$ & Exponent & Coefficient \\
\hline$s$ & 68.1600 & 0.00205 \\
$s$ & 10.2465 & 0.01596 \\
$s$ & 2.34648 & 0.07150 \\
$s$ & 0.673320 & 0.25547 \\
$s$ & 0.224660 & 0.27003 \\
$s$ & 0.082217 & 0.00953 \\
$s$ & 0.04 & 0.00988 \\
$s$ & 0.02 & -0.00342 \\
$z$ & 1.35 & -0.01710 \\
$z$ & 0.45 & -0.04320 \\
$z$ & 0.15 & -0.01389 \\
$z$ & 0.05 & -0.00095 \\
\hline \hline
\end{tabular}

a The first six s-type functions are from Ref. 17.

b The basis functions are symmetry adapted functions constructed from Cartesian-Gaussian functions of the given type.

functions centered at the origin.

In Tables III and IV we present our converged results for $\Sigma$ and $\Pi$ symmetries and compare them with those of Collins and Robb. ${ }^{10}$ Our results generally agree very well with those of Collins and Robb. Any discrepancies are probably due to the different target orbitals used. Collins and Robb use a target orbital constructed from Slater-type functions which probably gives a more accurate orbital than that constructed from our CartesianGaussian functions. It is interesting to note that in an earlier study, ${ }^{7}$ we used a target constructed from a smaller $5 s 2 z$ Cartesian-Gaussian basis set. Using the iterative Schwinger method with this target, we found that in the ${ }^{1} \Sigma_{u}$ channel there were discrepancies of $\sim 0.05 \mathrm{rad}$ in the converged eigenphase sums compared with the results of Collins and Robb. This large difference had not been evident in any of the other channels considered. We then performed a scattering calculation in which no exchange interaction was considered. This calculation with the direct potential only yielded excellent agreement with the equiva-

TABLE II. Scattering basis set. ${ }^{a}$

\begin{tabular}{llllll}
\hline \hline & \multicolumn{3}{c}{$\begin{array}{l}\text { Type of function for } \\
\text { the scattering } \\
\text { symmetry }\end{array}$} \\
$\Sigma_{g}$ & $\Sigma_{u}$ & $\Pi_{g}$ & $\Pi_{u}$ & Function center & Exponent \\
\hline$s$ & $s$ & $x$ & $x$ & Nuclei & 1.0 \\
$s$ & $s$ & $x$ & $x$ & Nuclei & 0.3 \\
$z$ & $z$ & $x z$ & $x z$ & Nuclei & 1.0 \\
$z$ & $z$ & $x z$ & $x z$ & Nuclei & 0.3 \\
$s$ & $z$ & $x z$ & $x$ & Bond midpoint & 1.0 \\
\hline \hline \\
\hline
\end{tabular}

TABLE III. Converged eigenphase sums for $\Sigma$ symmetries in $e^{-}-\mathrm{H}_{2}{ }^{+}$scattering.

\begin{tabular}{ccrrr}
\hline \hline \multicolumn{2}{c}{ Symmetry } & $k=0.2$ & $\begin{array}{c}\text { Momentum } \\
k=0.5\end{array}$ & $k=1.0$ \\
\hline${ }^{1} \Sigma_{\boldsymbol{g}}$ & $L M^{\mathrm{a}}$ & -0.366 & -0.377 & -0.352 \\
& $C R^{\mathrm{b}}$ & -0.363 & -0.384 & -0.350 \\
${ }^{3} \Sigma_{g}$ & $L M$ & 0.235 & 0.235 & 0.243 \\
& $C R$ & 0.230 & 0.233 & \\
${ }^{1} \Sigma_{u}$ & $L M$ & 0.349 & 0.401 & 0.519 \\
\multirow{2}{*}{${ }^{3} \Sigma_{u}$} & $C R$ & 0.359 & 0.412 & \\
& $L M$ & 1.408 & 1.296 & 1.074 \\
\hline \hline
\end{tabular}

${ }^{a}$ Results of the present study.

${ }^{b}$ Results from Ref. 10 .

lent calculation performed by Collins and Robb. This somewhat anomalous behavior in the ${ }^{1} \Sigma_{u}$ channel prompted us to try the more accurate $8 s 4 z$ target which then gave very good agreement in this channel. It is believed that this strong dependence on the target orbital is indicative of resonant-like scattering in the ${ }^{1} \Sigma_{u}$ channel.

The iterative procedure used in the present study was found to converge very rapidly. In Table $\mathrm{V}$ we present a representative calculation showing how the eigenphases converged. The higher partial-wave eigenphases were not accurately obtained using only the discrete basis set in the zeroth iteration result. However, these ergenphases are quickly corrected in the first iteration since they are Born dominated.

All integrals were computed using numerical quadrature as is described els ewhere. ${ }^{4,18}$ The integrals were evaluated on a grid of 780 points extending out to 66.2 a.u. All basis functions and the target orbital were expanded up to $l=13$. The exchange and direct integrals were then computed

TABLE IV. Converged eigenphase sums for $\Pi$ symmetries in $e^{-}-\mathrm{H}_{2}{ }^{+}$scattering.

\begin{tabular}{clrrr}
\hline \multicolumn{2}{c}{ Symmetry } & $k=0.2$ & $\begin{array}{c}\text { Momentum } \\
k=0.5\end{array}$ & $k=1.0$ \\
\hline \multirow{2}{*}{${ }^{1} \Pi_{g}$} & $L M^{\mathrm{a}}$ & 0.042 & 0.042 & 0.049 \\
& $C R^{\mathrm{b}}$ & 0.045 & 0.045 & 0.054 \\
${ }^{3} \Pi_{g}$ & $L M$ & 0.094 & 0.122 & 0.183 \\
& $C R$ & 0.097 & 0.128 & 0.194 \\
${ }^{1} \Pi_{u}$ & $L M$ & -0.344 & -0.330 & -0.281 \\
& $C R$ & -0.347 & -0.331 & -0.273 \\
${ }^{3} \Pi_{u}$ & $L M$ & 0.141 & 0.137 & 0.107 \\
& $C R$ & 0.154 & 0.150 & 0.119 \\
\hline \hline
\end{tabular}

${ }^{a}$ Results of the present study.

${ }^{b}$ Results from Ref. 10. 


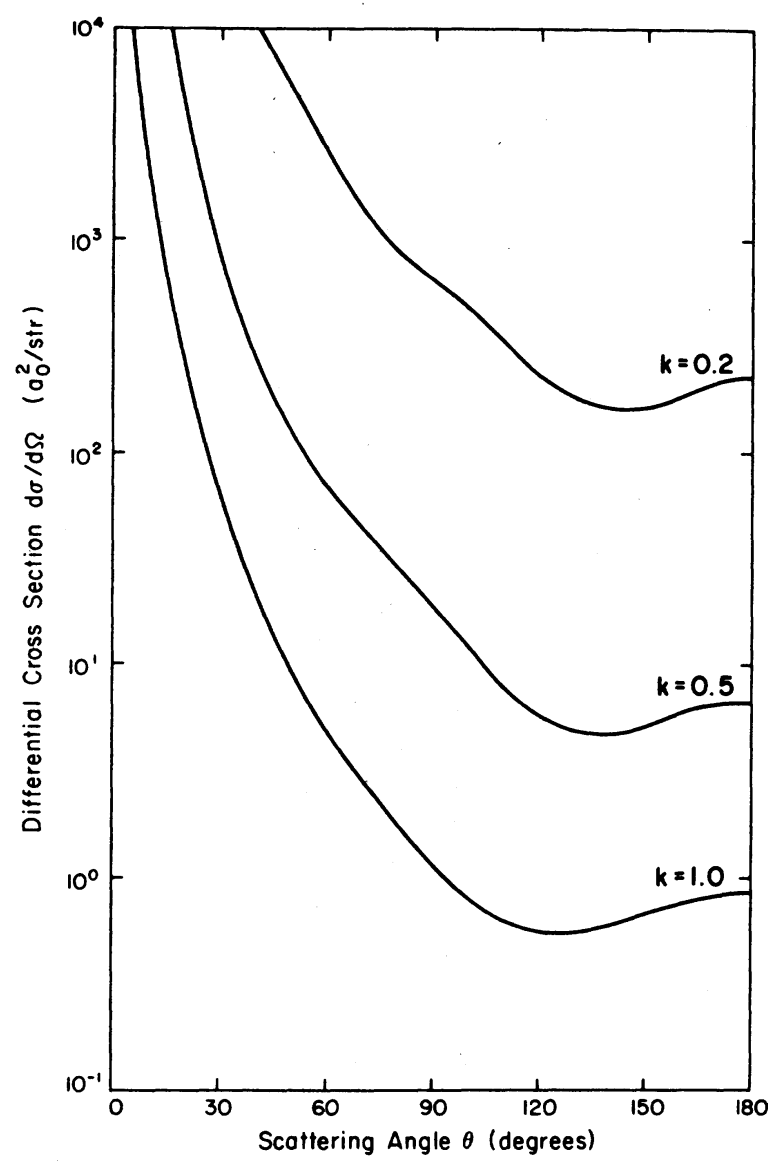

FIG. 1. Spin-averaged elastic DCS for $e^{-}-\mathrm{H}_{2}{ }^{+}$collisions.
TABLE V. Convergence of eigenphases using the iterative Schwinger variational method. ${ }^{a}$

\begin{tabular}{ccrrrr}
\hline \hline \multirow{2}{*}{$\begin{array}{c}\text { Iteration } \\
\text { number }\end{array}$} & 0 & 2 & \multicolumn{1}{c}{ Eigenphase $^{\mathrm{b}}$} & \multicolumn{1}{c}{6} & Sum \\
\hline 0 & -0.497 & 0.055 & -0.000 & 0.000 & -0.443 \\
1 & -0.488 & 0.083 & 0.019 & 0.006 & -0.379 \\
2 & -0.487 & 0.084 & 0.019 & 0.006 & -0.377 \\
$C R^{\mathrm{c}}$ & -0.497 & 0.088 & 0.019 & 0.006 & -0.384 \\
\hline \hline
\end{tabular}

${ }^{2}$ The results given are for ${ }^{1} \Sigma_{\mathrm{g}}$ scattering in $e^{-}-\mathrm{H}_{2}{ }^{+}$at $k=0.5$ a.u.

b These values of $l$ correspond to the principal component of the given eigenphase.

${ }^{\mathrm{c}}$ Results from Ref. 10.

exactly with no further truncations in $l$. We have computed partial-wave solutions up to $l_{p}=7$. This truncation of the sum in $l_{p}$ in general gives eigenphase sums converged to better than $1 \%$. Cutting off the $l_{p}$ sum does represent an approximation to the total wave function $\Psi_{\vec{k}}$, however, each individual partial-wave function $\psi_{k l m}$ which is included in the calculation will still be obtained exactly within the static-exchange approximation if the iterative solution converges. In Fig. 1 we present the spin-averaged DCS obtained at the three energies considered here. These curves clearly show the dominance of Coulomb scattering at low angle and the effects of scattering due to the short-range potential at large angles.

\section{RESULTS FOR PHOTOIONIZATION OF $\mathrm{H}_{2}$}

We use the electron-molecular-ion scattering wave functions obtained here to study the photoionization of $\mathrm{H}_{2}$ in its ground state. We have used the method outlined in our earlier paper ${ }^{4}$ to obtain the photoionization cross section. The fixed-nuclei photoionization cross section is obtained in both the dipole-length and dipole-velocity approximations.

The initial-state wave function $\Psi_{i}\left(\vec{r}_{1}, \vec{r}_{2}\right)$ used in these calculations is a Hartree-Fock wave function. The initial state is thus of the form

$$
\Psi_{i}\left(\overrightarrow{\mathrm{r}}_{1}, \overrightarrow{\mathrm{r}}_{2}\right)=\phi_{1 \sigma}\left(\overrightarrow{\mathrm{r}}_{1}\right) \phi_{1 \sigma}\left(\overrightarrow{\mathrm{r}}_{2}\right)\left(\frac{\alpha(1) \beta(2)-\beta(1) \alpha(2)}{2^{1 / 2}}\right) \text {. }
$$

The one-electron orbital $\phi_{1 \sigma}$ was constructed from a $5 s 2 z$ Cartesian-Gaussian basis given by Watson $e t$ al..$^{5}$ The Hartree-Fock energy for $\mathrm{H}_{2}$ in this basis set is -1.1330 a.u. The final-state wave function is taken to be the electron-ion scattering wave function where the target orbital is fixed as the $\phi_{1 \sigma}$ orbital of $\mathrm{H}_{2}$. Thus the final states are of the form

$$
\Psi_{f, \overrightarrow{\mathrm{r}}}\left(\overrightarrow{\mathrm{r}}_{1}, \overrightarrow{\mathrm{r}}_{2}\right)=\left(\frac{k}{2}\right)^{1 / 2}\left[\phi_{1 \sigma}\left(\overrightarrow{\mathrm{r}}_{1}\right) \Psi_{\overrightarrow{\mathrm{r}}}^{(-)}\left(\overrightarrow{\mathrm{r}}_{2}\right)+\Psi_{\overrightarrow{\mathrm{k}}}^{(-)}\left(\overrightarrow{\mathrm{r}}_{1}\right) \phi_{1 \sigma}\left(\overrightarrow{\mathrm{r}}_{2}\right)\right]\left(\frac{\alpha(1) \beta(2)-\beta(1) \alpha(2)}{2^{1 / 2}}\right) .
$$

The differential dipole oscillator strengths are then computed in either the length or velocity form using

$$
\left(\frac{d f}{d E}\right)_{L}=\sum_{\mu} \frac{2}{3} E \int d \Omega_{\hat{k}}\left|\left\langle\Psi_{i}\left|\gamma_{\mu}\right| \Psi_{f, \hat{k}}\right\rangle\right|^{2}
$$

or

$$
\left(\frac{d f}{d E}\right)_{V}=\sum_{\mu} \frac{2}{3} \frac{1}{E} \int d \Omega_{\hat{k}}\left|\left\langle\Psi_{i}\left|\nabla_{\mu}\right| \Psi_{f, \hat{k}}\right\rangle\right|^{2},
$$


ionization cross section is then given by

$$
\sigma(E)=\frac{2 \pi^{2}}{c}\left(\frac{d f}{d E}\right)
$$

The initial scattering basis set in these calculations was the same basis set we used for $e^{-}-\mathrm{H}_{2}^{+}$ scattering and is given in Table II. The rate of convergence in these photoionization calculations was similar to that obtained in $e^{-}-\mathrm{H}_{2}^{+}$scattering. In Table VI we give an example which shows the rate of convergence for a selected energy in the ${ }^{1} \Sigma_{u}$ symmetry. Both the eigenphase sums and the cross sections were converged to three decimal places by the second iteration for all energies considered in this study. In Fig. 2 we present the converged photoionization cross sections for $\mathrm{H}_{2}$ in both the length and velocity forms. We also compare the present calculated cross section with some experimental results.$^{19,20}$ Since we have not treated the vibrational motion in the $\mathrm{H}_{2}-\mathrm{H}_{2}{ }^{+}$system, we have only compared our calculated results with experimental results which correspond to photon energies for which the sum of all FranckCondon factors for the open vibrational channels of $\mathrm{H}_{2}^{+}$is close to unity. For the $\mathrm{H}_{2}-\mathrm{H}_{2}^{+}$system this corresponds to photon energies greater than $\sim 18 \mathrm{eV}{ }^{21,22}$ The equivalence of the dipole-length and -velocity forms of the photoionization cross section is a necessary but not a sufficient condition for the exact solution. ${ }^{21}$ Thus, we may use this difference to estimate the minimum possible error in the calculation. For the photonionization of $\mathrm{H}_{2}$, as shown in Fig. 2, the length and velocity forms bracket the experimental results except at the lowest energy. Thus in this case the difference between the length and velocity forms gives a good estimate to the true error in the calculation.

\section{CONCLUSION}

In this study we have extended the iterative Schwinger variational method to include electronmolecular-ion collisions. For the $e^{-}-\mathrm{H}_{2}^{+}$system

TABLE VI. Convergence of eigenphase sums and cross sections using the iterative Schwinger variational method to compute photoionization cross sections of $\mathrm{H}_{2}{ }^{2}$

\begin{tabular}{cccc}
\hline \hline \multirow{2}{*}{$\begin{array}{c}\text { Iteration } \\
\text { number }\end{array}$} & \multirow{2}{*}{$\begin{array}{c}\text { Eigenphase } \\
\text { sum }\end{array}$} & \multicolumn{2}{c}{$\sigma(\mathrm{Mb})^{\mathrm{b}}$} \\
Length & Velocity \\
\hline 0 & 0.181 & 4.59 & 2.62 \\
1 & 0.213 & 4.62 & 2.64 \\
2 & 0.213 & 4.62 & 2.64 \\
\hline \hline
\end{tabular}

a The results given are for ${ }^{1} \Sigma_{u}$ scattering at $k=0.4287$ or $E=18.9 \mathrm{eV}$.

${ }^{b}$ In megabarns $\left(10^{-18} \mathrm{~cm}^{2}\right)$.

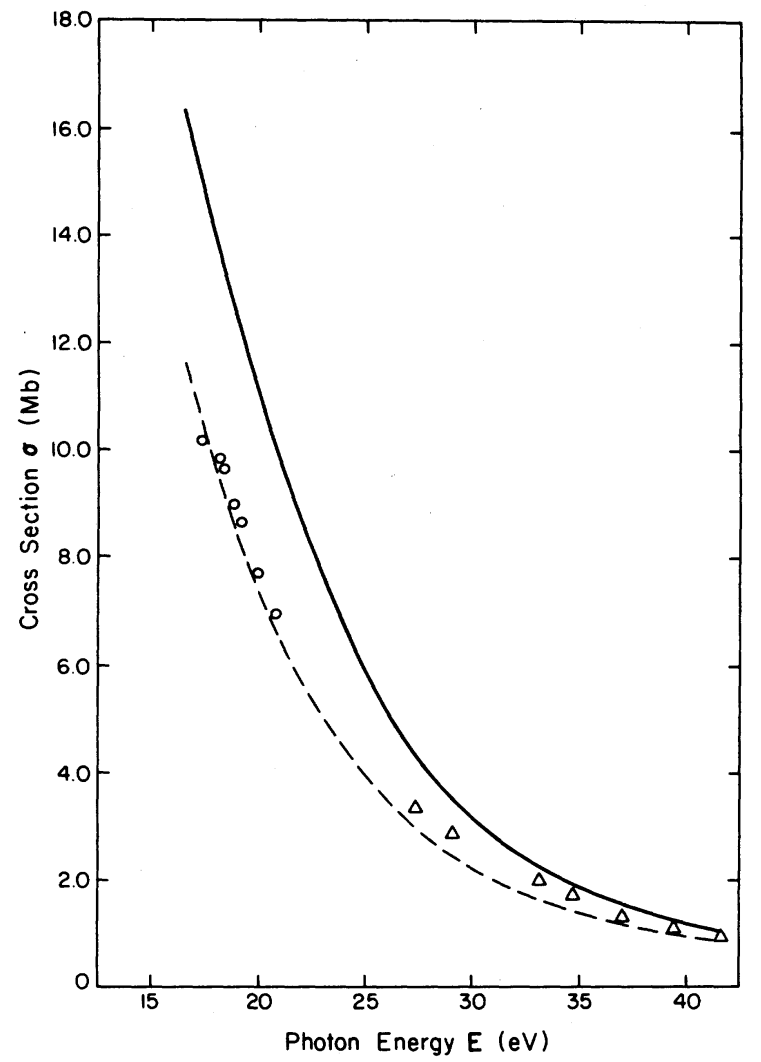

FIG. 2. Total photoionization cross section of $\mathrm{H}_{2}$ in megabarns: — static-exchange dipole length; --static-exchange dipole velocity; $O$ experimental points from Cook et al. (Ref. 19); $\Delta$ experimental points from Samson and Carins (Ref. 20). The ionization potential for $\mathrm{H}_{2}$ was taken to be $16.4 \mathrm{eV}$.

we found rapid convergence of the iterative scheme The resulting eigenphases are in close agreement with the accurate static-exchange results of Collins and Robb. ${ }^{10}$ We have also shown that the photoionization cross sections obtained using the method presented here agree well with the observed cross sections. The application of the iterative Schwinger method to the photoionization of $\mathrm{N}_{2}$ and $\mathrm{CO}_{2}$ is in progress.

\section{ACKNOWLEDGMENTS}

This work was supported by the National Science Foundation under Grant No. CHE79-15807 and was supported in part by the National Resource for Computation in Chemistry under a grant from the National Science Foundation and the Basic Energy Sciences Division of the United States Department of Energy under Contract No. W-7405-ENG-48. We would like to thank Dr. Derek Robb for many helpful discussions and for providing us with results prior to publication. One of us (R.R.L.) acknowledges the support of a National Science 
Foundation Predoctoral Fellowship. The research reported in this paper made use of the DreyfusNSF Theoretical Chemistry computer which was funded through grants from the Camille and Henry
Dreyfus Foundation, the National Science Foundation grant No. CHE78-20235, and the Sloan Fund of the California Institute of Technology.
'See, for example, Proceedings of the First A silomar Conference on Electron-Molecule and Photon-Molecule Collisions, Pacific Grove, 1978, edited by Thomas Rescigno, Vincent McKoy, and Barry Schneider (Plenum, New York and London, 1979).

${ }^{2}$ D. K. Watson and V. McKoy, Phys. Rev. A $\underline{20}, 1474$ (1979).

${ }^{3}$ R. R. Lucchese and V. McKoy, J. Phys. B $\underline{12}, 421$ (1979).

${ }^{4}$ R. R. Lucchese and V. McKoy, Phys. Rev. A 21,112 (1980).

${ }^{5}$ D. K. Watson, R. R. Lucchese, V. McKoy, and T. N. Rescigno, Phys. Rev. A 21, 738 (1980).

${ }^{6}$ R. R. Lucchese, D. K. Watson, and V. McKoy, Phys. Rev. A 22 , 421 (1980).

? For some preliminary results using discrete basis functions, see R. R. Lucchese and V. McKoy, Phys. Scr. 21, 366 (1980).

${ }^{8}$ R. R. Lucchese, G. Raseev, and V. McKoy (unpublished).

${ }^{9}$ D. K. Watson, V. McKoy, and T. N. Rescigno (unpublished).
${ }^{10} \mathrm{~L}$. Collins and D. Robb (private communication).

${ }^{11}$ A. Temkin and K. V. Vasavada, Phys. Rev. 160, 109 (1967).

${ }^{12}$ A. Temkin, K. V. Vasavada, E. S. Chang, and A. Silver, Phys. Rev. 186, 57 (1969).

${ }^{13}$ B. R. Tambe and B. Ritchie, J. Chem. Phys. 68,3595 (1978).

${ }^{14}$ W. H. Miller, J. Chem. Phys. 50, 407 (1969).

${ }^{15}$ S. K. Adhikari and I. H. Sloan, Phys. Rev. C 11, 1133 (1975).

${ }^{16}$ V. B. Belyaey A. P. Podkopoyev, J. Wrzecionko, and A. L. Zubarev, J. Phys. B 12, 1225 (1979).

${ }^{17}$ S. Huzinaga, J. Chem. Phys. 42, 1293 (1965).

${ }^{18}$ A. W. Fliflet and V. McKoy, Phys. Rev. A 18,2107 (1978).

${ }^{19}$ G. R. Cook and P. H. Metzger, J. Opt. Soc. Am. 54, 968 (1964).

${ }^{20}$ J. A. R. Samson and R. B. Cairns, J. Opt. Soc. Am. 55, 1035 (1965).

${ }^{21}$ H. P. Kelly, Chem. Phys. Lett. 20, 547 (1973).

${ }^{22}$ P. H. S. Martin, T. N. Rescigno, V. McKoy, and W. H. Henneker, Chem. Phys. Lett. 29, 496 (1974). 